glühendes Glas mit der Pfeife aus dem Ofen und drückte es mit Gewalt und rasch gegen den verletzten Theil des Fufses. Beim Zurückziehen blieb der Splitter an der weichen Glasmasse hängen, ohne dafs der Fufs durch die Hitze im Mindesten gelitten hatte.

\title{
Ueber die flüchtigen Säuren des Harns;
}

von $G$. Städeler.

Wie es öfters gelingt aus den Zerselzungsproducten, die man künstlich aus einem organischen Körper hervorbringt, eine wahrscheinliche Vorstellung von seiner eigentlichen Zusammensetzungsweise zu bekommen, so sucht man auch aus den Bestandtheilen der Excrete des lebenden Organismus, die als Endproducte der, das Blut und die Organe constituirenden Materien betrachtet werden müssen, eine Kenntnifs von der Constitution dieser Blutbilder und der chemischen Processe, die mit den Erscheinungen des Lebens in nothwendigem Zusammenhange siehen, zu erlangen. Vielfach sind defshalb die Absonderungsstoffe der Gegenstand chemischer Untersuchungen gewesen, doch sind die flüchtigen Bestandtheile derselben bis jetzt fast ganz unberücksichtigt geblieben, obwohl ihre Kenntnils von nicht geringerer Bedeutung für die Beantwortung jener wichtigen Fragen ist, wie die Kenntnifs der nicht flüchtigen Bestandtheile. Aus diesem physiologischen Gesichtspunkte habe ich eine nähere Untersuchung der bis jetzt noch fast ganz unbekannten flüchtigen Bestandtheile des Harns unternommen.

Nach einer Angabe von Berzelius soll Buttersäure im Harn vorkommen, die auch von Lehmann, jedoch als seltener Bestandtheil desselben, angenommen wird. Ich habe diese 
Säure nichl mil einiger Sicherheit nachweisen können, und im Kuhharn, den ich hauptsächlich zu meinen Versuchen anwandte, findet sich unter den Körpern, die unzweideutig den Character von Säuren besitzen, als Hauptbestandtheil eine flüchtige ölförmige Säure, die sich zunächst der Oenanthsäure anschliefst, indem sie zu dieser in demselben Verhältnils steht wie die Angelikasäure zur Valeriansäure und die Akrylsäure zur Propionsäure.

Ganz besonders überraschend war aber das Auftreten der Phenylsïure (Carbolstiure), die ich nicht allein im Harn der Herbivoren, sondern auch im Menschenharn aufgefunden habe. Sie kommt zugleich mil einem anderen Körper vor, dessen Eigenschaften nur wenig von denen der letzteren abweichen, sich aber durch einen grölseren Kohlensloff - und Wassersloffgehalt davon unterscheidet.

Da diese flüchtigen Körper einen sehr kleinen Theil des Harns ausmachen, so wurde dadurch auch die Untersuchung sehr ersclıwert. Die vorliegende Arbeit erstreckt sich aus diesem Grunde hauptsächlich nur auf den Harn von Kühen; da mir dieser am leichtesten zugängig war, und ich aufserdem für eine andere Arbeit gleichzeilig gröfsere Mengen von Hippursäure bereiten konnte. Einige Versuche, die ich mit dem Harn von Pferden und Menschen angestellt habe, kann ich jetzt nur anhangsweise miltheilen, werde dieselben aber, sobald es meine Zeil erlaubt, weiter ausdehnen und dabei ganz besonders auf den Harn im gesunden und krankhaften Zustande Rücksicht nehmen.

\section{1) Untersuchung des Kuhkarns.}

Frischer Kuhharn*) wurde mit Kalkhydrat vermischt, nach einmaligem Aufkochen vom überschüssigen Kalk abgegossen

*) Es wurden zu dieser Untersuchung etwa 80 Pfd. Morgenharn von Kühen angewandt, welche den Tag uher auf die Weide gingen und Morgens 
und bei Siedhitze auf etwa $\frac{1}{8}$ eingedampft. Das Filtrat wurde darauf bei guter Abkühlung mit Salzsäure bis zur stark sauren Reaction vermischt und die ausgeschiedene Hippursäure nach etwa 12 Stunden von der Mutterlauge getrennt.

Bei der Destillation derselben ging eine milchige Flüssigkeit von äufserst widrigem Geruch über, aus der sich gewöhnlich einige zähe Oeltropfen von gelber oder auch grünlicher Farbe abschieden.

Durch wiederholte Rectification gelang es die im Wasser gelösten flüchtigen Körper zu condensiren und es wurde endlich ein ölförmiges, schwach gelbliches Liquidum crhalten, welches gröfstentheils in dem mit übergegangenen Wasser niedersank.

Bei einer vorläufigen Prüfung konnte Phenylsäure sowohl durch die Reaction mit Eisenchlorid, als auch durch die blaue Farbe, welche ein mit Salzsäure benetzter Fichtenspahn damit annahm, nachgewiesen werden, doch war ein sehr widerlicher Geruch, ganz ähnlich dem des frischen Kuhharns vorherrschend, so dafs der Geruch der Phenylsäure nicht deullich wahrgenommen werden konnte. Aufserdem ging aus der verschiedenen

und Abends mit Heu, Stroh und Kleie gefüttert wurden. Nie habe ich daraus Benzoësäure, sondern immer Hippursäure erhalten, auch dann, wenn der Harn ohne Zusatz von Kalk bei Siedhitze abgedampft wurde. Nach zwei quantitativen Versuchen beträgt der Gehalt an Hippursäure nahe 1,5 pC. Ein Uebergang der Hippursäure in Benzoësïure während des Abdampfens scheint mir, früheren Angaben entgegen, niemals stattzufinden. Reine Hippursäure kaun z. B. Tage laug mit Wasser geliocht werden, olne dafs die geringste Zersetzung eintritt. Nur verdunntere Säuren und Alkalien veranlassen bei Sicdhitze rasch die Zersetzung der Hippursäure in Benzoësäure und Glycin, und da beim Kochen des Harns mit Kalkmilch die Bildung brauner harzähnlicher Körper veranlafst wird, die der Hippursäure hartnäckig anhängen und ibre Reinigung erschweren, so bietet Gregory's Darstellungsweise dieser Säure durchaus keinen Vortheil dar. 
Schwere des Destillats und aus dem Verhalten gegen Kalilauge, welche einen 'Theil des Oels ungelöst liefs, hervor, dafs es ein Gemenge von verschiedenen Körpern sey.

Das gewonnene Oel sammt dem wässerigen Destillat wurde defshalb mit einer gewogenen Menge Kalihydrat bis zur stark alkalischen Reaction vermischt und der Destillation unterworfen, wodurch ein schwach gelbgefärbtes leichtes $0 \mathrm{el}$ von durchdringendem Geruch erhalten wurde.

Dieses $\mathrm{Oel}$, dessen Geruch am besten mit dem eines Gemenges von Rosmarin- und Origanumöl verglichen werden kann, ist nicht ursprünglich in Harn vorhanden, sondern scheint sich erst durch Einwirkung von Kali aus dem unangenehm ricchenden Körper zu bilden. Da die Lösung nach einigen Tagen einen schwachen Ammoniakgeruch zeigte, so wurde sie mit etwas verdünnter Schwefelsäure der Destillation unterworfen, wobei das gebildete Ammoniaksalz zurückgehalten wurde. Das Destillat reagirte vollkommen neutral. Beim Erhitzen des Oels mit Natronkalk entwickelte sich viel Ammoniak und beim Vermischen mit concentrirler Schwefelsäure löste es sich unter Erhitzung mit tief weinrother Farbe, wurde aber allmälig heller und auf Zusatz von Wasser farblos. Die wässerige Lösung wurde durch basisch - essigsaures Bleioxyd nicht gefällt und gab auch mit Eisenchlorid keine Färbung.

So wünschenswerth eine nähere Untersuchung dieses stickstoffhaltigen Oels seyn mufste, so war sie doch der geringen Ausbeute wegen unmöglich.

Da die Abscheidung der Hippursäure aus dem eingedampften Harn mit Salzsäure geschehen war, so mulste unter den an Kali gebundenen Säuren auch Salzsäure vorhanden seyn, und ebenfalls war Benzoësäure, gebildet durch Zersetzung von Hippursäure, zugegen. Um diese letzteren zurückzuhalten, wurde ร vom angewandten Kali mit Schwefelsäure gesätligt, und so 
lange destillirt, bis in dem Destillat durch basisch-essigsaures Bleioxyd kein Niederschlag mehr entstand.

Die erhaltene Flüssigkeit, die jetzt ganz den Geruch der Phenylsäure hatte, wurde wiederholt mil Kochsalz neuen Destillationen unterworfen, bis der grölste Theil der Säuren ölförmig erhalten war und nur noch eine geringe Menge einer wässerigen Lösung übrig blieb.

Da dieselbe stark sauer reagirte, so konnte dic Oelschicht nicht allein aus Phenylsäure bestelıen. Zur Abscheidung dieser sauer reagirenden Körper wurde defshalb das Destillat mit kohlensaurem Natron gesättigt und 12 Slunden hindurch häufig geschüttelt. Die Oelschicht hatle sich dadurch vermindert und konnte durch Ausziehen mil Aether von den Natronsalzen getrennt werden.

a. Untersuchung der Säuren, welche das kohlensaure Natron nicht zersetzen. Von der ätherischen Lösung wurde der Aether bei gelinder Wärme abdestillirt und der Rückstand. zur Abscheidung der letzten Spuren desselben mit concentrirter Kalilauge der Destillation unterworfen. Es wurde dabei noch etwas von dem oben erwähnten indifferenten Oel erhalten, welches nur äufserst schwierig von den sauren Körpern getrennt werden kann. Die zurückbleibende Verbindung derselben mit Kali wurde darauf mit Kalibicarbonat zersetzt und das übergehende Product, welches elwa $25 \mathrm{Grm}$. betragen mochte, mil geschmolzenem Chlorcalcium längere Zeit in Berührung gelassen und endlich über Chlorcalcium rectificirt.

Das Destillat wurde in fünf Portionen aufgefangen. Schon bei $100^{\circ}$ zeigte sich ein Anflug im Retortenhalse und als das Thermometer auf $120^{\circ}$ gestiegen war, entstand eine schwache Blasenbildung, wobei gleichzeitig ölige Tropfen und eine milchige Flüssigkeit übergingen. Diese erste Porlion enthielt so viel Wasser, dafs sie sich bald in zwei Schichten trennte, die zweite war zwischen $170^{\circ}$ bis $180^{\circ}$ übergegangen, die dritte 
(wobei die Kugel des Thermomelers nur von heilsem Dampf umgeben war) zwischen $180^{\circ}$ bis $195^{\circ}$, die vierte bei $195^{\circ}$ und bei der fünften stieg das Thermometer auf fast $200^{\circ}$, wobei das Uebergehende sich etwas bräunte.

Die Hauptmenge des Destillats wurde zwischen $180^{\circ}$ bis $195^{\circ}$ erhalten. Die äufseren Eigenschaften waren übereinstimmend mit denen der Phenylsäure. Es war farblos, roch in hohem Grade dem Castoreum ähnlich, erzeugte nach wenigen Augenblicken einen weifsen Fleck auf der Haut, von welchem sich nach einigen Tagen die Haut abschuppte, färbte bei Gegenwart von Salzsäure Fichtenholz intensiv blau *), wurde in wässeriger Lösung durch Eisenchlorid blau gefärbt und nach einiger Zeit schied sich ein heller Niederschlag ab. Neutrales essigsaures Bleioxyd veranlafste keine Fällung, basisches Bleisalz dagegen bewirkte sogleich einen weilsen voluminösen Niederschlag. Aus der Darstellungsweise geht ferner hervor, dals dieser Körper mit laustischem Alkali eine Verbindung bildet, dafs er aber nicht die kohlensauren Salze zerlegen kann, vielmehr durch Kohlensäure aus seinen Verbindungen ausgetrieben wird.

Diefs Verhalten stimmt vollkommen mit dem der Phenylsäure überein, dagegen wurde der Siedepunkt für die Hauptmenge des Destillats höher gefunden**); auch ist es mir nicht

*) Diese Reaction kommt am schönsten zum Vorschein, wenn man einen Fichtenspahn mit einer wässerigen Lüsung von Phenylsäure tränkt, ihn darauf einen Augenblick in verdünnte Salzsäure taucht und den Sonnenstrahlen aussetzt. Fr wird dann in wenigen Augenblicken tiof blau gefäıbt und die Farbe widersteht, wie schon ll unge beobachtet hat, sehr hartnäckig der Einwirkung des Chlors. Sie wird zwar heller, kommt aber sogleich wieder zum Vorschein, wenn der Spahn in verdünnte Salzsüure getaucht wird.

**) Nach La urent siedet die Phenylsäure bei $187-188^{\circ}$. 
gelungen, durch Abkühlung mit Eis und Kochsalz eine Krystallisation zu bewirken.

Ueber diese Abweichungen konnte die Analyse zunächst Aufschlufs guben, sie wurde defshalb mit der zweiten, drillen und vierten Portion gemacht. Die Verbrennung geschah mit Kupferoxyd und Sauerstoff.

II. 0,3545 Grm. gaben 0,9735 Grm. Kohlensäure und 0,2395 Grm. Wasser.

III. 0,403 Grm. gaben 1,1393 Grm. Kohlensäure und 0,2685 Grm. Wasser.

IV. 0,297 Grm. gaben 0,840 Grın. Kohlensäure und 0,202 Grm. Wasser.

0,270 Grm. gaben, mit Natronkalk geglüht, 0,012 Plalin.

Der Stickstoffgehalt mufste bei der letzten Analyse aus dem Platin bestimmt werden, weil dem Chlorplatinanmonium nach dem Waschen mit Alkohol und Aether feine, Linien lange weilse Prismen beigemengt waren.

Aus diesen Analysen ergiebt sich folgende procentische Zusammenselzung :

\begin{tabular}{|c|c|c|c|}
\hline Kohlenstoff & $\begin{array}{c}\text { II. } \\
\mathbf{7 4 , 9 0}\end{array}$ & $\begin{array}{c}\text { III. } \\
77,10\end{array}$ & $\begin{array}{l}\text { IV. } \\
77,14\end{array}$ \\
\hline Wasserstoff & 7,51 & 7,40 & 7,56 \\
\hline $\begin{array}{l}\text { Sauerstoff } \\
\text { Stickstoff }\end{array}$ & 17,59 & 15,50 & $\begin{array}{r}14,67 \\
0,63\end{array}$ \\
\hline & 100,00 & 100,00 & 100,00 \\
\hline
\end{tabular}

Der Slickstoffgehalt rührt offenbar von einem geringen Rilckhalt an indifferentem Oel her, welches, wie schon bemerkt, sich nur schwierig vollkommen abscheiden Iäfst. Die Gegenwart desselben wird auch noch dadurch bewiesen, dafs beim Vermischen der obigen Destillate mit concentritter Schwefelsäure eine hellweinrothe Färbung eintritt, die nach einiger Zeit ganz verschwindet. 
Wollte man diesem stickstoffhaltigen Körper auch einen nicht ganz unbedeutenden Einflufs auf das Resultat der Analysen sugestehen, so würde dasselbe doch noch immer wenig Uebereinstimmung mit der Zusammensetzung der Phenylsäure zeigen. Diese besteht nämlich in 100 Theilen aus :

$$
\begin{array}{rllrr}
12 & \text { Aeq. } & \text { Kohlenstoff } & 900 & 76,60 \\
6 & \rightarrow & \text { Wasserstoff } & 75 & 6,38 \\
2 & \Rightarrow & \text { Sauerstoff } & 200 & 17,02 \\
\cline { 3 - 4 } & & & 1175 & 100,00 .
\end{array}
$$

Die obigen Analysen zeigen im Wasserstoffgehalt eine $\mathbf{A b}$ weichung von mehr als 1 pC., die bei der zweiten Portion des Destillats theilweise von hygroscopischem Wasser herriihrt, wodurch auch der Kohlenstoff um einige Procente niedriger ausfällt. Diese Erklärung kann aber nicht bei den anderen Analysen Anwendung finden; es mufs daher der Phenylsäure ein Körper beigemengt seyn, der reicher sowohl an Kohlenstoff als auch an Wasserstoff ist.

Es ist mir in der That geglückt einige wesentliche Unterschiede zwischen beiden Körpern aufzufinden, doch sind meine Bemühungen, sie zu trennen, bis jetzt gänzlich fruchtlos geblieben. Der kohlenstoffreichere Körper, den ich Taurylsäure nennen werde, unterscheidet sich von der Phenylsäure nicht nur durch einen höheren Siedepunkt, sondern auch durch sein Verhalten gegen concentrirte Schwefelsäure. Wäbrend die Phenylsäure eine Verbindung damit eingeht, die selbst nach Monaten noch fluissig bleibt, bildet die, mit einem gleichen Volum Schwefelsäure vermischte Taurylsäure eine Verbindung, die nach kurzer Zeit anfängt sich in zarten weilsen Dendriten, die allmälig zu kugeligen Massen zusammenwachsen, auszuscheiden. Die vierte Portion des Destillats war so reich an Taurylsäure, dafs sie nach kurzer Zeit gänzlich erstarrte, die dritte dagegen erstarrte nur theilweise. Als ich die erstarrte Masse auf eine Gypsplatte legte, um die anhängende Schwefel- 
säure und Phenylschwefelsäure davon einsaugen zu lassen, nahm sie rasch Feuchtigkeit aus der Luft auf und entzog sich durch Zerfliefsen jeder weiteren Untersuchung.

Ich kann daher die Zusammensetzung der Taurylsäure nicht durch eine gut stimmende Analyse nachweisen; wahrscheinlich aber ist es, dafs sie dieselbe Zusammenselzung hat, welche man bisher für das Kreosot*) angenommen hat, dals ihre $\mathrm{Zu}-$ sammensetzung also durch die Formel $\mathrm{C}^{14} \mathrm{H}^{8} \mathrm{O}^{2}$ ausgedrückt wird. Sie wäre demnach nit dem Anisol isomer, von welchem

*) Die obige Formel wurde aus Ettling's Analysen (Annal. d. Pharm. VI, 209), die einzigen, welche bis jetzt vom Kreosot bekannt sind, abgeleitet. Berechnet man dieselben aber nach den jetzigen richtigeren Atomgewichten, so weichen die gefundenen und die berechnelen Mengen von Kohlenstoff und Wasserstoff so sehr von einander ab, dafs diese Formel unmõglich für das Kreosot beibehalten werden kann. Bekanntlich ist das Kreosot, welches man jetzt kăuflich bezieht, gewölnnlich nichts anderes als Phenylsäure. Nach einigen Versuchen, die ich aber mit Kreosot, welches sich durch den eigenthümlichen Rauchgeruch auszeichnete, angestellt habe, scheint auch dieses nichts anderes als eine unreine Phenylsäure zu seyn. Das Verhalten gegen Fichtenholz ist wenigstens ganz dasselbe und auch mit Eisenchlorid giebt die Lósung anfangs eine blaue Farbe, die aber rasch durch Bräunung verdeckt wird.

Nimmt man an, dafs Ettling eine reine Substanz analysirt habe, so würden seine Analysen viel besser mit der Formel $\mathrm{C}^{\prime 2} \mathrm{H}^{\prime} \mathrm{O}^{\text {? }}$ übereinstimmen, wie folgende Zusammenstellung zeigt :

Ettling erhielt von :

I. 0,429 Grm. Kreosot 1,191 Kohlensäure und 0,301 Wasser.

II. $0,520, \quad$ " $, 421 \quad 0,364$ "

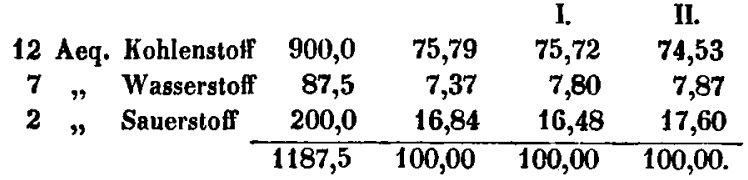

Eutling fand den Wasserstoff zwar höher als der aufgestellten Formel entspricht, doch findet man einen solchen Ueberschufs an Wasserstoff bei älteren Aualysen sehr häufig. 
sie sich aber schon durch den weit höher liegenden Siedepunkt unterscheidet.

Die Rechnung giebt :

\begin{tabular}{|c|c|c|c|}
\hline \multicolumn{2}{|c|}{4 Aeq. Kohlenstoff } & 1050 & 77,77 \\
\hline 8 & Wasserstoff & 100 & 7,40 \\
\hline 2 & Sauer'stoff & 200 & 14,83 \\
\hline & & 1350 & 100,0 \\
\hline
\end{tabular}

Die oben gefundene Zusammensetzung des dritten und vierten Destillationsproductes stimmt unter der Voraussetzung, dafs ein Gemenge von Taurylsäure und Phenylsäure analysirt wurde, sehr gut mit dieser Rechnung überein, und ich hielt es defshalb für unerläfslich, die Gegenwart der letzteren noch auf andere Weise, als durch die angeführten Reaclionen nachzıweisen.

Da die Phenylsäure sehr leicht durch Salpetersäure in $\mathrm{Pi}-$ krinsalpetersäure verwandelt wird, so wurde die zweite Portion des Destillats, welche bei 170 bis $180^{\circ}$ übergegangen war, und also am wenigsten Taurylsäure enthalten mufste, mit Salpetersäure von 1,3 spec. Gew. ubergossen, wobei eine heftige Einwirkung staltfand und sich ein brauner, harzähnlicher Körper abschied, der sich beim Kochen vollsländig wieder auflöste. Beim Erkalten krystallisirte eine Säure in grofsen Blältern, deren Menge zwar nicht für eine Analyse hinreichend war, die aber nach Form, Farbe, Geschmack und dem Verhalten des Kalisalzes nichts anderes als Pikrinsalpetersäure seyn konnte. Die von den Krystallen abgegossene Mutterlauge liefs beim Vermischen mit Wasser eine gelbe, elwas zusammenklebende Substanz fallen, die wahrscheinlich von einer Nitroverbindung der beigemengten 'Taurylsäure herrührte.

lch habe ferner den Theil der Schwefelsäureverbindung, welcher beim Verınischen der dritten Portion des Destillats mit einem gleichen Volum Schwefelsäure nicht erstarrte, von der festen Masse (Taurylschwefelsäure) abgegossen und durch Ko- 
chen mit kohlensaurem Bleioxyd eine Bleiverbindung darzustellen versucht. Das neutral reagirende Filtrat hinterlief́s beim Verdampfen im luftleeren Raume eine weifse, amorphe Masse, die anhaltend zwischen 100 bis $110^{\circ}$ getrocknet wurde. Anfangs entwich nur Wasser, später gab sich auch der Geruch der Phenylsïure zu erkennen. Sobald dieser Punkt eingetreten war, wurde die Verbindung als wasserfrei betrachtet und der Bleigehalt bestimint.

$0,929 \mathrm{Grm}$. gaben 0,512 schwefelsaures Bleioxyd $=55,11 \mathrm{pC}$.

Die Formel des phenylschwefelsauren Bleioxyds $=\dot{\mathrm{PbS}}+\ddot{\mathrm{S}}$, $\mathrm{C}^{12} \mathrm{H}^{5} \mathrm{O}$ verlangt $54,80 \mathrm{pC}$.

Es wird also durch diese Versuche die Gegenwart der Phenylsäure in dem analysirlen Gemenge aulser Zweifel gesetzt.

b. Untersuchung der Säuren, welche das kohlensaure Natron zersetzt hatten. Die Lösung der Natronsalze, welche durch Behandeln nit Aether von den bereits abgehandelten Körpern getrennt war, wurde zır Entfernung des aufgelösten Aethers eingedampft, dann mil Schwefelsäure zerselzt und der Destillation unterworfen.

Das Destillat, welches einen eigenthümlichen, der Buttersäure elwas ähnlichen Geruch halle, trennte sich in zwei Schichten. Die untere bildele ein farbloses, schweres, ölförmiges Liquidum, die obere war eine Auflösung dieser Säuren in Wasser und röthete stark das Lackmuspapier.

Zur weiteren Untersuchung wurde zuerst die wässerige Schicht mit kohlensaurem Baryt gekocht und die erhaltene alkalische Lösung theilweise verdunstet. Am anderen Tage waren schöne, büschelförmig verwachsene, platte glänzende Prismen angeschossen, die dem capronsauren Baryt sehr ähnlich waren. Die ganze Ausbeule belrug nach dem Trocknen bei $120^{\circ}$ $0,1457 \mathrm{Grm}$., welche bei slarkem Erhitzen schmolzen und $0,0515 \mathrm{Grm}$. kohlensauren Baryt hinterliefsen.

Die von diesen Krystallen getrennte Flüssigkeit wurde im 
luftleeren Raume über Schwefelsäure weiter verdunstet. Erst als die Lauge bedeutend eingeengt war, zeiglen sich von Neuem Krystalle. Da diese noch etwas von dem früher angeschossenen Salz :enthalten konnten, so wurden sie fortgenommen und erst in den zunächst anschiefsenden Krystallen der Barytgehalt bestimmt. Es waren sehr kleine weifse Prismen, die beim Erhitzen nicht zusammenschmolzen, sondern einen kohlensauren Baryl hinterliefsen, der ganz die Form der Krystalle halle. 0,3425 Grm. gaben 0,1735 kohlensauren Baryl.

Durch noch weiteres Verdunslen wurde endlich ein driltes Salz in sehr undeutlichen Krystallen erhalten, die zur Befreiung von der syrupförmigen Mutlerlauge auf Löschpapier gelegt und nach dem Trocknen bei $120^{\circ}$ geglüht wurden. Sie unterschieden sich durch ibre Schmelzbarkeit wesentlich von der vorigen Krystallisation. 0,1253 Grm. gaben 0,0717 kolulensauren Baryt.

Da sich aus dem Angefiuhrten kein weiterer Schluls ziehen liefs, als dafs die Barylsalze mindestens drei Säuren enthielten, so wurde auch die noch übrige ülförmige Säure zur Bereilung von Barytsalzen angewandt.

$0,113 \mathrm{Grm}$. der ersten Krystallisation gaben 0,040 kohlensauren Baryt.

0,0572 Grm. der zweiten Krystallisation gaben 0,0205 kohlensauren Baryt.

0,3514 Grm. der dritten Krystallisation gaben 0,1765 kohensauren Baryt.

$0,0585 \mathrm{Grm}$. der vierten Krystallisation gaben 0,0295 kohlensauren Baryt.

Die fünfte Krystallisation, welche ebenso wie die dritte und vierte nicht schmelzbar war, wurde zur Darstellung eines Silbersalzes benutzt und die geringe Menge der zurïckbleibenden Mutterlauge hinterliefs nach dem Verdunslen ein Salz, welches schmelzbar war und elwas über 41 pC. Baryt enthielt. 
Aus der folgenden Zusammenstellung werden leicht dio zusammengehörenden Salze erkannt werden :

I. Bereilung. 1. Krystallisation (schmelzhar) cuthiell 27,46 pC. Ba

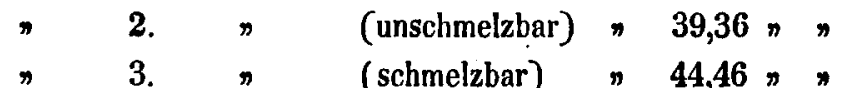

II. Bereilung. 1. "

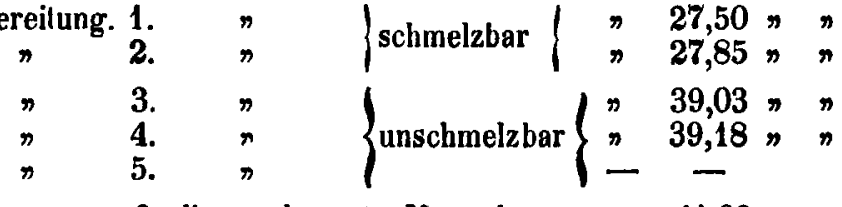

6. die verdunstete Mutterlauge $\gg 41,00$,

Den Hauptbestandtheil dieses Gemenges macht die Säure aus, deren Barytsalz etwas über 39 pC. Baryt enthält. Da die ganze Ausbeule an Säuren nur einige Grm. betrug, so war es mir allein bei dieser letzteren möglich, durch Zersetzung des Barytsalzes mit salpetersaurem Silberoxyd ein Silbersalz darzustellen, welches zur Bestimmung der Elementarbestandtheile der Säure dienen konnte. Ich werde mich defshalb zunächst zu dieser Säure wenden.

Das Silbersalz bildet ein weilses, sich am Licht nicht veränderndes Pulver.

$0,1072 \mathrm{Grm}$, bei $110^{\circ}$ getrocknet, hinterliefs nach dem Glühen 0,049 regulinisches Silber.

0,380 Grm. gaben 0,493 Kohlensäure und 0,1602 Wasser. Hieraus berechnet sich folgende Zusammensetzung :

\begin{tabular}{|c|c|c|c|c|}
\hline 1 Aeq. & Silberoxyd & 1450,0 & $\begin{array}{c}\text { berechnet } \\
49,36\end{array}$ & $\begin{array}{c}\text { gefundor } \\
49,11\end{array}$ \\
\hline 14 & Kohlenstoff & 1050,0 & 35,74 & 35,38 \\
\hline ग & Wasserstoff & 137,5 & 4,68 & 4,68 \\
\hline » & Sauerstoff & 300,0 & 10,22 & 10,83 \\
\hline & & $\overline{2937,5}$ & 100,00 & 100,00 \\
\hline
\end{tabular}

Demnach besteht die wasserfreie Säure aus $\mathrm{C}^{14} \mathrm{H}^{11} \mathrm{O}^{3}$, was auch vollkommen mit der Zusammensetzung der analysirten Barylsalze übereinstimmt : 


\begin{tabular}{lccccc} 
& & & \multicolumn{3}{c}{ gefunden } \\
\cline { 3 - 6 } Baryt & 958,0 & 39,18 & 39,36 & 39,03 & 39,18 \\
Säure & 1487,5 & 60,82 & 60,64 & 60,97 & 60,82 \\
\hline & 2445,5 & 100,00 & 100,00 & 100,00 & $100,00$.
\end{tabular}

Da es nun keinem Zweifel unterliegt, dafs in den Salzen 1 At. Wasser der Säure durch 1 At. Base ersetzt ist, so mufs die Zasammensetzung der freien Säure, für welche ich den Namen Damalursäure (gebildet aus $\delta \alpha \dot{\mu} u \lambda \iota s$ und ovjov) vorschlage, durch die Formel $\dot{\mathrm{H}}, \mathrm{C}^{14} \mathrm{H}^{11} \mathrm{O}^{9}$ ausgedruickt werden.

Sie besteht in 100 Theilen aus :

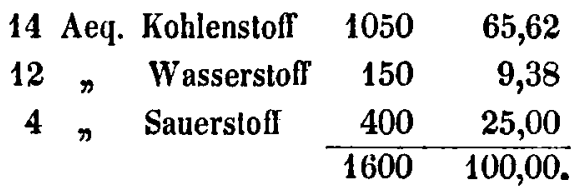

Die Damalursäure hat einen eigenthümlichen, wie mir scheint, der Valeriansäure nicht unähnlichen Geruch, sie ist etwas schwerer als Wasser und bildet mit basisch essigsaurem Bleioxyd einen weifsen Niederschlag, den man unter dem Mikroscop aus feinen, kugelförmig zusammengewachsenen Prismen beslehend erkennt.

Von der Oenanthsäure unterscheidet sie sich dadurch, dafs sie 2 Aeq. Wassersloff weniger enthält, ferner durch das spec. Gewicht, durch den Geruch, durch die Form und das Verhalten des Barylsalzes beim Erhitzen und durch die Bleiverbindung, da das önanthsaure Bleioxyd, nach Tilley's Angabe, als citronengelbes Pulver niederfällt.

Die Säure, welche sich in zunächst gröfster Quantitäl in dem Gemenge der Säuren findet, ist die, deren Barytverhindung zuerst aus der Lösung anschiefst, und sich schon durch die Schmelzbarkeit vom damalursauren Baryt unterscheidet.

Nach drei Beslimmungen enthält dieses Salz 27,46, 27,50 und 27,85 pC. Baryt. Da die Lösung dessellen, ebenso wie 
die Lösung des damalursauren Baryls, das gerölhele Lackmuspapier bläut, so kann es kein saures Salz seyn, und das Atomgewicht der Säure würde also, wenn man dasselle aus dem Millel der drei Barylbeslimmungen bercchnet, 2513,0 betragen.

Ein nicht sehr abweichendes Atomgewicht hat die Cocinsäure $(2562,5)$, die aber, ganz so wie die zunächst sich anschlielsenden felten Säuren, bei gewöhnlicher Temperatur fest ist, während die Säure aus dem Harn sich aus dem Barytsalz auf Zusaiz von Salzsäure in schweren ölförmigen, nicht erstarrenden Tropfen abscheidet.

Sie kann also nicht in die Reihe der felten Säuren gehören, und ist eine bis jetzt nicht gekannte Säure, die sich in der Zusanmenselzung vielleicht ähnlich zur Cocinsäure verhält, wie die Damalursäure zur Oenanthsäure. Um ihre Abstammung anzudeuten halte ich für dieselbe den Namen Damolsäure für passend.

Was endlich die Salze, deren Barytgehalt über $40 \mathrm{pC}$. steigt, anbetriff, so werden diese nur als Gemenge von damalursaurem Baryt mit einem anderen Barytsalze belrachtet werden dürfen. Ob die Säure in diesem Salze aber Buttersäure, Valeriansäure oder ebenfalls cine noch unbekannte Säure ist, mufs ich für jetzt unentschieden lassen.

Es blieb noch nachzuweisen über, ob die oben erwähnten Körper präformirt im Harn enthalten sind, oder ob sie erst durch Einwirkung der zur-Abscheidung angewandten Reagentien gebildet wurden. Ich habe in dieser Beziehung einige Versuche angestellt.

$2500 \mathrm{Grm}$. Harn wurden bei ganz gelinder Wärme auf $\frac{1}{8}$ eingedampft, nach dem Erkalten mit verdünnter Schwefelsäure vermischt und die ausgeschiedene Hippursäure nach zwölfstündigem Stehen abfiltrirt. 
Die tiefbraune Multerlauge wurde mil Aether geschüttelt, die rothe ätherische Lösung abgehoben und der Aether verdunstet, worauf ein rothbraunes, in gröfserer Menge gesehen fast schwarzes, ölförmiges Liquidum zurückblieb.

Durch Schütteln mit einer Lösung von kohlensaurem Natron wurde ein Theil der sauren Körper gebunden, worauf ein anderer Theil mit Aether ausgezogen werden konnte. Die erhaltene rolbbraune ätherische Lösung wurde von Neuem in gelinder Wärme verdunstet und der Rückstand mil ganz wenig Wasser der Destillation unterworfen, wobei gelbe Tropfen übergingen, während im Kolben ein braunes Harz zurückblieb.

Das Destillat wurde durch Eisenchlorid blau gefärbt, dagegen war der Geruch der Phenylsäure durch den Geruch des indifferenten Oeles verdeckt; er kam aber deutlich zum Vorschein, als das letztere von den an Kali gebundenen Säuren durch Destillalion getrennt und die Phenyl- und Taurylsäure durch verdiunte Schwefelsäure in Freiheit geselzt wurde.

Die Nalronsalze wurden ebenfalls mit Schwefelsäure zersetzt und die Säuren mit Aelher ausgezogen. Die Lösung hinterliefs nach dem Verdunsten einen dunkelbraunen Rückstand, durch dessen Destillation mit Wasser ölförmige Tropfen erhallen wurden. Sie hatten den Geruch der drei beschriebenen Säuren, dem sich aber der bekannte widerliche Geruch des Kuhharns zugesellte. Am deutlichsten kam dieser Geruch zum Vorschein, wenn man einige Tropfen des Destillats auf der Haut verdunsten liefs.

Durch einfache Destillation des Kuhharns wurde ein unangenehm riechendes, ammoniakalisches Product erlualten, in welchem sich weder Phenylsäure noch Damalursäure nachweisen liefs.

Aus diesen Versuchen geht hervor, dafs die abgehandellen Säuren fertig gebildet, und zwar an ein Alkali gebunden im 
Harn vorkommen. Ob dagegen die ganze Menge der Damalursäure als solche irn Harn sich befindet, ist mir aus folgendem Grunde zweifelhaft : Als ich den Rückstand, welcher durch Zersetzung des phenylsauren und taurylsauren Kalis mit zweifach kohlensaurem Kali erhalten war, mil Schwefelsäure übersälligte und der Destillation unterwarf, ging noch eine Portion der letzteren Säuren über, aber gemengt mit einer geringen Menge einer anderen Säture, die das kohlensaure Natron zersetzle und Damalursäure zu seyn schien.

Dic einzige Erklärung, die ich für diese Thatsache $z u$ geben im Stande bin, ist die, dafs sich diese Säure aus einem anderen Körper durch Einwirkung von Kali gebildet hat, und dafs als ein zweites dabei auftretendes Product das indifferento stickstofthallige Oel zu betrachlen ist. Die Stammverbindung würde dann wahrscheinlich der übelriechende Körper des Harns seyn, da dieser ja, wie schon angeführt, bei der Reinigung der beschriebenen Producte fast vollständig verschwindet.

Dieser Körper würde demnach durch Säuren und Alkalien eine ganz ähnliche Zerselzung erleiden, wie sie von der Hippursäure bekannt ist, und in die neue Säure und das stickstoffhaltige Oel zerfallen, welches dann in Zusammensetzung und Eigenschaften dem Glycin nicht ganz fern stehen dürfte.

Eine quantitative Bestimmung der flüchligen Beslandtheile des Harns ist mir bis jelzt nicht möglich gewesen. In Betreff der relaliven Mengen würde die Taurylsäure obenan gestellt werden müssen, ihr folgen die Phenylsäure und die Damalursäure, die Damolsäure heträgt kaum ein Viertel der Danalursäure und in geringster Menge findet sich die Säure, deren Barytsalz mehr als 40 pC. Baryt enthält.

\section{2) Versuche mit Pferdeharn und Menschenharn.}

Als ich Pferdeharn mit Kalkhydrat abdampfte und nach der Zerselzung mit Salzsäure der Destillation unter warf, erhielt ich 
eine eben so grofse Menge flüchtiger ölförmiger Säuren, wie man sie aus einer gleichen Menge Kuhharn zu erhalten pflegt. Bei der weiteren Behandlung mit Kali, kohlensaurem Natron elc. war ebenfalls keine Verschiedenheit zu bemerken, so dats, obgleich analylische Belege fehlen, angenommen werden darf, dals die flüchtigen Säuren des Pferdeharns nicht von denen des Kuhharns verschieden sind.

Bei diesen Versuchen ist mir ein sonderbarer Umstand aufgeslo[sen, der lier milgelheilt $\mathrm{zu}$ werden verdient. Ich erhielt nämlich aus einer Quanlität Harn nur Spuren von Hippursäure, obgleich auch die Benzoësäure vollständig darin fehlte. So viel mir bekannt, ist ein solcher Fall bis jelzt nicht beobachlet worden. Der Harn war am Morgen von einem Pferde gesammelt, welches selten einen Tag im Stalle zubrachte und das, nach der Aussage des Besilzers, fast allein mit Hafer gefüllert wurde. Ich zweifelte anfangs, dals dieser Harn wirklich von Pferden herstamme, doch überzeugte ich mich bald durch die verhältnifsmäfsig grofse Ausbeute an flüchtigen Säuren, dafs dazu kein weiterer Grund vorhanden war.

Mit Menschenharn habe ich nur einen Versuch angestellt und habe darin auf gleiche Weise, wie im Kuh - und Pferdeharn, beide Gruppen von Säuren nachgewiesen *). Auch das aromatisch riechende Oel fehlte unter den flüchtigen Producten nicht, so dafs sich in Bezug auf die Qualität derselben keine bemerkenswerthe Abweichung vom Harn der Herbivoren zeigt; die Quantität dagegen ist sehr viel geringer, indem bei Anwendung von 3 Pfund Harn nichts weiter als eine sichere Nachweisung der verschiedenen Körpergruppen möglich war.

Bekanntlich hat Scharling ${ }^{*}$ ) aus Harn, der durch Frost

\footnotetext{
*) Siehe diese Annalen Bd. L, S. 172. d. Red.

**) Ebendaselbst Bd, XLII, S. 265.
} 
concentrirt war, mit Aether einen Körper ausgezogen, den er Omichmyloxyd nennt, und der im trocknen Zuslande dem Castoreum ähnlich riecht. Dafs dieses s. g. Omichmyloxyd keine einfache Verbindung, sondern ein Gemenge von verschiedenen Körpern ist, kann nicht bezweifelt werden, und da der Geruch des Castoreums nach Wöhler's *) Untersuchung von beigemengter Phenylsäure herrührt, so glaube ich annehmen zu dürfen, dafs auch diese Säure einen Gemengtheil von Scharling's Omichmyloxyd ausmachl.

Wirft man endlich noch einen Blick auf den Ursprung der beschriebenen Säuren, so kann kaum daran gezweifell werden, dafs die Damalursäure und Damolsäure als Umsetzungsproducte thierischer Substanzen zu betrachten sind, da ähnliche flüchtige Säuren auch im Schweils, in den Feltarten und unter den Oxydationsproducten des Albumins, Fibrins, Caseïns und des Leims aufgefunden worden sind.

Am unerwarletslen ist das Vorkommen von Phenylsäure und der ihr so ähnlichen Taurylsãure im lebenden Körper, zumal die erstere nach den Versuchen von $W \ddot{\text { h }} \mathrm{ler}$ und Frerichs**) entschieden giflig wirkt.

Ich selbst zweifelte anfangs an der Präexistenz dieser Süure; der Versuch, den ich in dieser Beziehung angestellt habe, spricht aber so unzweideutig dafür, dafs ich jetzt nur noch einige Worte uber ihre mögliche Abstammung hinzuzufügen hale.

Die Phenylsäure bildet sich bekanntlich leicht aus einigen Gliedern der Salicylgruppe, die ziemlich weit im Pflanzenreiche verbreitet zu seyn scheinen. Man kann also annehmen, dafs diese Slamıverbindungen dem Körper durch die Nahrung zuge-

*) Diese Annalen Bd. XLIX, S. 360 und Bd. LXVII, S. 360.

**) Diese Annalen Bd. LXV, S. 344. 
führt werden, und dafs die, durch Zersetzung derselben entstandene Phenylsäure sogleich durch die Nieren ausgeschieden und dadurch ibr schädlicher Einflufs beseitigt werde. Hierfür spricht auch die Beobachtung Lehmann's *), dafs nach dem Genufs von Salicin aus dem Harn mit Aether ein Körper ausgezogen werden kann, der durch Eisenoxydsalze gebläut wird, denn die normale Menge der Phenylsäure im Menschenharn scheint nicht so bedeutend zu seyn, dafs sie sich auf so einfache Weise in einer kleinen Quantität von Harn nachweisen liefse. L e hmann nimmt zwar an, dafs die genannte Reaction von salicyliger Säure herrührt, doch verhalten sich beide Säuren gegen Eisenoxydsalze so ähnlich, dafs dadurch eine Unterscheidung laum möglich ist.

Ganz besonders wird aber die aufgestellte Ansicht noch dadurch unterstülzt, dafs Wöhler in Castoreum neben Phenylsäure auch Salicin aufgefunden hat, welches offenbar aus der Nahrung der Bieber, die vorzugsweise aus Pappel- und Weidenrinde, also aus sehr salicinreichen Substanzen besteht, abstamınt. Man darf hieraus auch schliefsen, dafs der Harn der Bieber sehr reich an Phenylsäure seyn mufs; und da nach der Untersuchung von E. H. Weber ${ }^{* *}$ ) die Castorbeutel nichts anderes sind als sackförmig gefaltete Erweilerungen des Praepulium penis und clitoridis der Bieber, deren Construction das Eindringen des Harns zuläfst, so kar:a nicht daran gezweifelt werden, dafs sowohl die Phenylsäure, als auch das Salicin und die Benzoësäure, welche in Casloreum vorkommen, nicht in den Beuteln gebildet, sondern durch den Harn in dieselben eingeführt werden.

Das Castoreum wäre demnach nichts anderes als die mit Harnbestandtheilen gemengte Hautsalbe des Präputiums der Bieber,

*) Handwörterb. der Physiolog. II, 15.

*) Berichte d. k. säclis. Gesellsch. der Wissensch. II, 185. 
und die therapeutische Wirkung desselben möchte wobl schwerlich einem anderen Körper als der Phenylsäure darin zugeschrieben werden können.

So wohl begründet nun auch die Ansicht, dafs die Phenylsäure von Körpern aus der Salicylgruppe abstamme, ist, so scheint doch die Annahme, dafs sie allein aus diesen hervorgehe, nicht stalthaft zu seyn. Schlieper*) hat diese Säure, jedoch nur spurweise, unter den Oxydationsproducten des Leims aufgefunden, und da die Verwandlung, welche organische Stoffe im Organismus erleiden, gewöhnlich der Zersetzung durch oxydirende Materien ganz ähnlich ist, wofür die Verwandlung des Bittermandelöls und der Harnsüure Beispiele liefern, so ist es möglich, dafs die Phenylsäure auch ganz unabhängig von äufseren Einflüssen aus Bestandtheilen des Körpers, wohin zunächst die leimgebenden Gebilde zu rechnen wären, entstehen kann.

\section{Ueber die Theorie der Aetherbildung;} von Al. Williamson.

(Gelesen vor der British Association zu Edinburgh).

Wird Schwefelsäure mil Alkohol unter gewissen Umständen in Berührung gebracht, so geht in dem Alkohol eine neue Anordnung der Elemente vor sich, in deren Folge eine Spaltung in zwei Gruppen, in Aether und Wasser, stattfindet. Es ist bekannt, dafs der Procefs, in welchem diese Aenderung vor sich geht, in zwei verschiedenen Weisen dargestellt werden kann,

*) Diese Annalen Bd. LIX, S. 22. 